\author{
1 Балтийская международная академия, Рига, Латвия \\ 2 ФГБУН Институт вычислительной математики РАН, Москва, Россия \\ з Латвийский институт органического синтеза, Рига, Латвия \\ ${ }^{4}$ АНО «Центр биотической медицины», Москва, Россия
}

\begin{abstract}
Трансмембранный трафик электрогенных металлов (ЭМ) и в первую очередь ионов $\mathrm{Na}^{+}$тесно связан с биоэнергетикой клетки. С помощью метода количественной спектрометрии клеток эпидермиса (волосы) у здоровых лиц и ликвидаторов Чернобыльской аварии (окислительный/нитрозативный стресс) обнаружена сопряженность данных спектрометрии ЭМ в указанных группах. Было показано, что клетка, будучи открытой динамической системой, обнаруживает признаки самоорганизованной критичности (СК), что позволяет отнести гомеостаз ЭМ к СК-явлениям.
\end{abstract}

Ключевые слова: электрогенные металлы, редокс-статус, эпидермис, самоорганизованная критичность, окислительный/нитрозативный стресс

\title{
ELECTROGENIC METALS IN EPIDERMIS: CONNECTION WITH CELL BIOENERGETICS
}

\author{
V.I. Petukhov ${ }^{1,}$ E.V. Dmitriev ${ }^{2}$, L.K. Baumane ${ }^{3}$, A.V. Skalny ${ }^{4}$, Y.N. Lobanova ${ }^{4}$ \\ ${ }^{1}$ Baltic International Academy, Riga, Latvia \\ 2 Institute of Numerical Mathematics RAS, Moscow, Russia \\ ${ }^{3}$ Latvian Institute of Organic Synthesis, Riga, Latvia \\ ${ }^{4}$ Centre for Biotic Medicine, Moscow, Russia
}

We have found out in our previous studies that results of quantitative spectrometry of electrogenic metals (Ca, $\mathrm{K}, \mathrm{Na}$ ) in epidermal cells (hair) of healthy people and the Chernobyl liquidators (chronic oxidative/nitrosative stress) are conjugated. The nature of this "conjugation», like many intimate mechanisms of the metal-ligand homeostasis in the epidermis, remains unsolved. Transmembrane traffic of electrogenic metals (and especially the $\mathrm{Na}^{+}$ion) is directly related to cell bioenergetics. Therefore, intracellular bioenergetics processes in the membrane and in the mitochondrial respiratory chain, can determine homeostasis of electrogenic metals. It has been shown that in the cell, that is an open dynamic system, there are signs of self-organized criticality (SC). Thus we can classify electrogenic metal homeostasis as $S C$-phenomenon.

Key words: electrogenic metals, redox status, epidermis, self-organized criticality, oxidative/nitrosative stress

\section{ВВЕДЕНИЕ}

Гомеостаз металлов в эпидермальных клетках, как подтверждают многочисленные публикации на эту тему и наши собственные наблюдения [5, 11], можно изучать, используя для этой цели дериват эпидермиса (волосы). Это особенно ценно для массовых (скрининговых) исследований, позволяющих получить необходимое число проб для достоверного статистического анализа.

Такой подход был реализован в настоящей работе для подтверждения тесной связи электрогенных металлов (ЭМ) с биоэнергетикой клетки, а также для поиска свидетельств возможной принадлежности гомеостаза ЭМ к явлениям самоорганизованной критичности (СК).

Основанием для данного допущения могут служить уже известные факты: 1) генерация и поддержание электрохимического потенциала клетки за счёт постоянного и разнонаправленного трафика ЭМ через плазматическую мембрану; 2) участие ионов натрия $\left(\mathrm{Na}^{+}\right)$в клеточном энергообмене в качестве дополнительной к аденозинтрифосфату (АТР) конвертируемой «энергетической валюты»; 3) несостоятельность гипотезы о нормальном распределении данных количественной спектрометрии металлов, содержащихся в деривате эпидермиса (волосы) [5].
Живая клетка, будучи открытой динамической системой, зависит от энергии, поступающей извне. Однако без предварительной трансформации этой энергии в конвертируемые и удобные для борьбы с энтропией формы (ATP, $\mathrm{Na}^{+}, \mathrm{H}^{+}$) она не смогла бы найти своё рациональное применение. В этой связи регуляция внутриклеточного энергетического баланса (соответствие энерготрат их адекватному восполнению) приобретает несомненную актуальность.

Один из возможных теоретических подходов, помогающих успешному анализу регуляции энергообмена (причём не только в микро-, но и в макросистемах), предоставляет нам теория самоорганизованной критичности [1]. В рамках этой теории получают своё объяснение статистика катастроф (закон Гутенберга - Рихтера для землетрясений), численные закономерности роста городов и городского населения, а также частотного распределения слов в речи и литературном тексте (закон Ципфа), фрактальная геометрия, функционирование нейронных сетей головного мозга и другие события микро- и макромира, которые относят к так называемым СК-явлениям.

Наиболее наглядной моделью СК, ставшей своеобразным «брендом» этой теории, служит куча песка, формируемая при постоянном добавлении последнего в минимальных количествах. Динамические 
взаимоотношения между песчинками в этой куче, приводящие к «сходу лавин» различного калибра, характеризуются степенной связью между размерами лавин и их числом в заданном интервале измерений. Обнаружение степенной зависимости, на которую никак не влияет масштаб изучаемого объекта (критерий фрактальности), помогает выявить СК-феномены в различных динамических системах.

Гомеостаз ЭМ в клетках регенерирующих тканей (эпидермис) имеет непосредственную связь с клеточной энергетикой. При этом ключевые механизмы гомеостатического контроля над уровнем металлов следует, по-видимому, искать в особенностях внутриклеточного энергообмена, т. к. нельзя исключить наличие общих регуляторных механизмов, обладающих признаками самоорганизованной критичности.

Поэтому представляет интерес обнаружение возможной степенной связи между содержанием ЭМ в эпидермисе (спектрометрия) и числом индивидов, принадлежащих заданному интервалу количественных оценок, что и стало целью настоящей работы. Разумеется, такие исследования должны проводиться на достаточно большом статистическом материале.

\section{МАТЕРИАЛЫ И МЕТОДЫ}

С помощью методов математической статистики были проанализированы результаты атомно-эмиссионной спектрометрии волос на определение содержания $\mathrm{Na}, \mathrm{K}, \mathrm{Ca}$, полученные в Центре биотической медицины (Москва), у 10297 здоровых лиц, жителей Москвы и Риги (5160 мужчин и 5137 женщин) в возрасте от 2 до 85 лет. Забор материала и все исследования проводились при обязательном согласии испытуемых.

Пусть имеется выборка $x_{1}, \ldots, x_{n}$ случайной величины $x \sim F_{x}(t)$, и по этой выборке построен вариационный ряд $x_{(1)} \leq x_{(2)} \leq \ldots \leq x_{(n)}$. Точечная оценка медианы $m$ распределения $\left(F_{x}(m)=0,5\right)$ - выборочная медиана - представляется в виде

$$
\tilde{m}=\left\{\begin{array}{ll}
x_{(k)}, & n=2 k-1 ; \\
\frac{\left(x_{(k)}+x_{(k+1)}\right)}{2}, & n=2 k .
\end{array} .\right.
$$

Данная оценка дает числовую оценку медианы, однако ничего не говорит о ее точности и достоверности. Определяемая по конечной выборке из генеральной совокупности, выборочная медиана по сути является случайной величиной и даже при сколь угодно большом n может сильно отличаться от истинного значения медианы. Интервальные оценки позволяют указать пределы, в которых с некоторой вероятностью $\rho=1-\alpha$ лежит искомый параметр. Поиск интервальной оценки медианы $P\left(m_{l o}<m<m_{u p}\right) \geq 1-\alpha$ подразумевает поиск распределения выборочной медианы. При $n>50$ можно принять, что $m_{l o}=x_{(\lambda)}$ и $m_{u p}=x_{(n-\lambda+1)}$, где $\lambda=0,5\left(n-\tau_{1-\alpha / 2} \sqrt{n}-1\right), \tau_{1-\alpha / 2}-$ квантиль стандартного нормального распределения $N(0,1)$ [2].

Альтернативный подход к построению доверительных интервалов выборочной медианы основан на методе повторных выборок. Точечная оценка, сделанная по одной выборке с неизвестным распределением, представляет собой единственную реализацию случайной величины. Чтобы получить представление о ее распределении, необходимо иметь ансамбль реализаций. Для этого используют различные искусственные методы. В нашей работе мы использовали метод, известный как «бутстрэппинг». В его основе лежит основное положение математической статистики - выборочное распределение. Метод может быть сформулирован следующим образом.

Пусть имеющаяся выборка объема n образует генеральную совокупность с дискретным распределением $P\left(\zeta=x_{i}\right)=1 / n$. Из данной генеральной совокупности производится $k$ случайных выборок с возвращением объема $n$. В результате получаются выборки, состоящие из элементов исходной выборки, при этом, поскольку имеет место выбор с возвращением, могут быть повторяющиеся элементы. Поскольку выбор делается случайным образом, и генеральная совокупность при этом не меняется, то повторные бутстрэп-выборки можно считать независимыми.

Стандартный подход, используемый для построения доверительного интервала для оцениваемого параметра при заданном $\alpha$, состоит в следующем.

1. На основе повторных бутстрэп-выборок строится ансамбль $\left\{\tilde{m}_{b o o t}(i)\right\}_{i=1}^{k}$ оценок параметра т.

2. С троится вари ационный ряд $\tilde{m}_{(1)} \leq \tilde{m}_{(2)} \leq \ldots \leq \tilde{m}_{(k)}$

3. Строится эмпирическая функция процентилей. Каждая $\tilde{m}_{(i)}$ трактуется как перцентиль $\frac{i-1 / 2}{k} \times 100$.

В остальных точках проводится линейная интерполяция. Таким образом, нижний и верхний доверительный интервалы задаются, соответственно, соотношениями

$$
\begin{aligned}
& m_{l o}=\tilde{m}_{(i)}+\left(\tilde{m}_{(i+1)}-\tilde{m}_{(i)}\right)(n \alpha / 2-i+1 / 2), \\
& m_{u p}=\tilde{m}_{(j)}+\left(\tilde{m}_{(j+1)}-\tilde{m}_{(j)}\right)(n(1-\alpha / 2)-j+1 / 2) . \\
& \text { Если } \frac{\alpha}{2}<\frac{1-1 / 2}{n}, \text { то } m_{l o}=\tilde{m}_{(1)} \text { и } m_{i p}=\tilde{m}_{(k)} .
\end{aligned}
$$

\section{РЕЗУЛЬТАТЫ И ОБСУЖДЕНИЕ}

Дееспособность человеческих митохондрий, главных продуцентов АТР, не одинакова на протяжении всей жизни индивида. Снижение АТР-продукции в раннем детстве, когда процесс окончательного формирования митохондриальной сети ещё не завершён, сменяется нарастанием энергопродукции в период полового созревания с достижением максимума в наиболее работоспособном возрасте (18-45 лет). При старении происходит прогрессирующее уменьшение числа митохондрий, которое связывают с деструктивным действием активных форм кислорода (АФК) и азота (АФА), что в конечном итоге приводит к снижению интенсивности синтеза АТР [9].

Если же принять во внимание участие ионов $\mathrm{K}^{+}$и $\mathrm{Na}^{+}$в клеточном энергообмене, то представляет интерес не только возможные изменения в содержании этих металлов в эпидермисе, связанные с возрастом, но и сопоставление данных спектрометрии металлов 
с активностью митохондрий в разные возрастные периоды. Поэтому по результатам спектрометрии волос нами была найдена медиана уровня Na и К в разных возрастных группах. Результаты представлены в таблице 1 и на рисунках 1, 2.

Таблица 1 Возрастная динамика уровня К и Nа в эпидермисе

\begin{tabular}{|c|c|c|c|}
\hline Возраст, годы & Медиана К, мкг/г & ди bоot, низ & дИ boot, верх \\
\hline $60-85$ & 121,4 & 87,97 & 161,5 \\
\hline $50-59$ & 104,3 & 95,37 & 117,56 \\
\hline $30-49$ & 56,06 & 53,4 & 58,46 \\
\hline $20-29$ & 38,18 & 35,7 & 41,04 \\
\hline $10-19$ & 54,4 & 41,2 & 84,8 \\
\hline $2-9$ & 376,88 & 231,9 & 972,26 \\
\hline Возраст, годы & Медиана Na, мкг/г & ди boot, низ & ди boot, верх \\
\hline $60-85$ & 293,9 & 222,9 & 346,7 \\
\hline $50-59$ & 228,1 & 211,8 & 241,6 \\
\hline $30-49$ & 117,71 & 113,4 & 122,6 \\
\hline $20-29$ & 82,2 & 78,1 & 86,2 \\
\hline $10-19$ & 88,9 & 67,1 & 137,6 \\
\hline $2-9$ & 324,9 & 233 & 580,7 \\
\hline
\end{tabular}

Примечание. ДИ - доверительные интервалы; boot - bootstrap-метод.
Возрастная динамика содержания Na и К в эпидермисе демонстрирует два периода значимого повышения уровня металлов: первый - в возрасте от 2 до 9 лет; второй - после 50 лет (рис 1, 2). Чем объяснить эти изменения в содержании ЭМ?

Нам представляется оправданным связывать их происхождение с уже упоминавшейся недостаточной продукцией АТР в детском и старческом возрасте. Натриевый потенциал в качестве конвертируемой энерговалюты способен, по-видимому, компенсировать недостаток АТР в эти возрастные периоды. При этом повышенная продукция АФК/АФА у людей пожилого и старческого возраста должна приводить к более интенсивной работе мембранных помп (в первую очередь $\mathrm{Na}^{+} / \mathrm{K}^{+}$-АТРаз) за счёт окислительной модификации и/или S-нитрозилирования этих белков. В этом случае АФК/АФА играют важную роль сигнальных молекул, способствующих сохранению в клетке необходимого уровня энергии и как следствие - увеличению продолжительности жизни индивида.

Решающее значение для продления жизни клетки и организма в целом имеет, по-видимому, не столько наличие или отсутствие прооксидантного сдвига в его редокс-статусе, сколько сохранение энергетического потенциала на уровне клетки и всего организма [9]. И если этой цели служит способный к взаимотрансформации с другими энерговалютами (АТР и $\mathrm{H+)}$ натриевый потенциал, в реализации которого

\section{K}

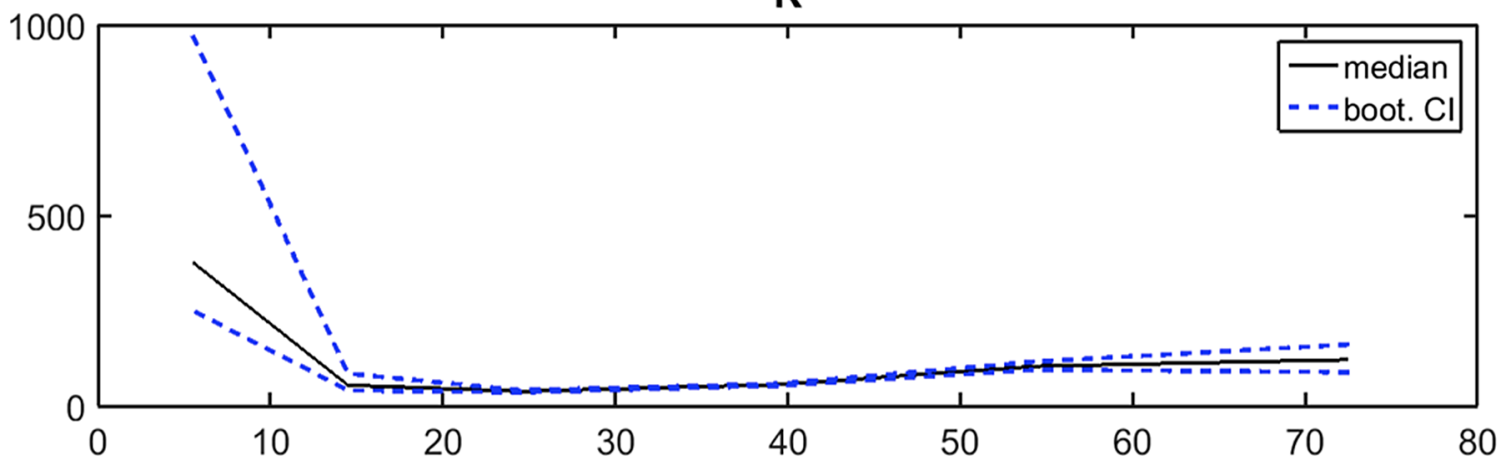

Рис. 1. Содержание калия в клетках эпидермиса в зависимости от возраста у здоровых лиц ( $n=10297)$ : по оси абсцисс возраст (годы); по оси ординат - уровень К (мкг/г); пунктирной линией обозначены ДИ (bootstrap-метод).

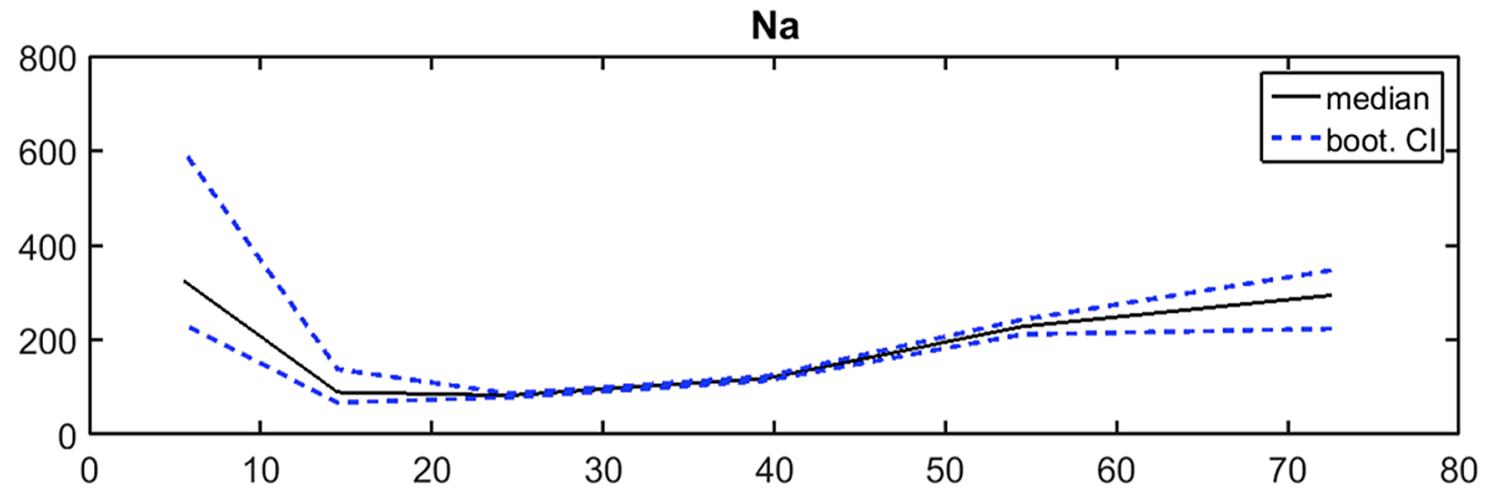

Рис. 2. Содержание натрия в клетках эпидермиса в зависимости от возраста у здоровых лиц ( $n=10297)$ : по оси абсцисс - возраст (годы); по оси ординат - уровень Na (мкг/г); пунктирной линией обозначены ДИ (bootstrap-метод). 
участвуют АФК/АФА и $\mathrm{Na}^{+} / \mathrm{K}^{+}$-АТРаза, то прооксидантный сдвиг и возрастание уровня натрия в клетке по мере старения организма вполне ожидаемы.

Это обстоятельство реабилитирует известную способность АФК/АФА накапливаться в старческом возрасте и заставляет усомниться в универсальности теории свободнорадикального старения, в которой свободным радикалам отводится роль главных факторов старения [3].

Пока не очень ясно, что стимулирует работу $\mathrm{Na}^{+}$/ $\mathrm{K}^{+}$-АТРаз у детей, хотя имеются сообщения о прооксидантном сдвиге в редокс-статусе детей в возрасте 7-11 лет, который авторы связывают с дефицитом натуральных антиоксидантов и адаптационными нарушениями [7].

Связь гомеостаза ЭМ с биоэнергетикой делает вероятным участие самоорганизованной критичности в контроле над внутриклеточным содержанием ЭМ. Поэтому представлял интерес характер связи между уровнем ЭМ (данные спектрометрии) и числом индивидов в заданных интервалах измеряемых значений ЭМ. Обнаружение степенной зависимости между этими параметрами (критерий СК) позволило бы отнести гомеостаз ЭМ к разряду СК-явлений.

Как показали наши предыдущие исследования, содержание кальция в волосах у женщин было значимо больше, чем у мужчин [5]. Поэтому статистическая обработка данных спектрометрии для Са проводилась отдельно для мужчин и женщин. Результаты исследования представлены на рисунках 3,4 .

На рисунках 3,4 , выполненных в двойном логарифмическом масштабе, представлена модель - плотность степенного распределения (число столбиков $=20$ ):

$$
y(x)=\frac{a b^{a}}{x^{a+1}} \text { при } x \geq b .
$$

Подгонка линейной функции $\ln y=A \ln x+B$, где $A=-a-1 ; B=\ln a+a \ln b$; методом наименьших квадратов последних 9 столбиков. При этом прямая визуально хорошо аппроксимирует данные в логарифмическом масштабе, однако в линейном масштабе такой вариант не является оптимальным решением с точки зрения наименьших квадратов.

В качестве гипотезы мы предлагаем следующую трактовку полученных данных.

На всех представленных кривых (рис. 3 , 4) можно обнаружить отрезки разной величины, практически совпадающие с прямой линией, которые, согласно теории СК, свидетельствуют о степенном характере связи между исследуемыми параметрами (уровень ЭМ и число индивидов) и/или критическом состоянии в функционировании АТРаз (с параметром ветвления $\sigma=1$ ).

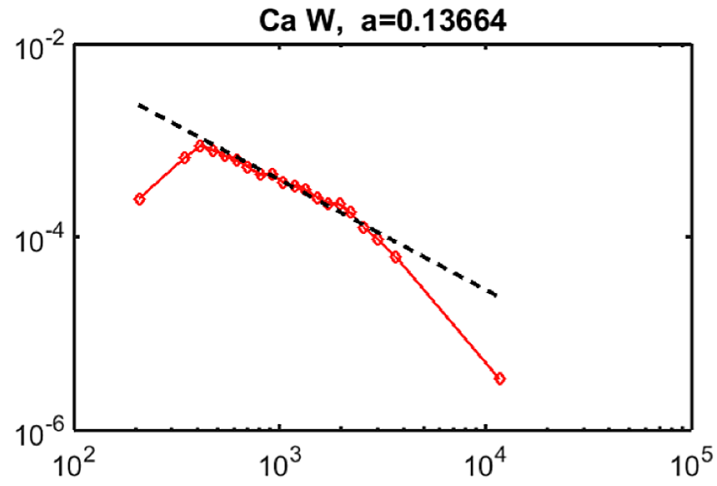

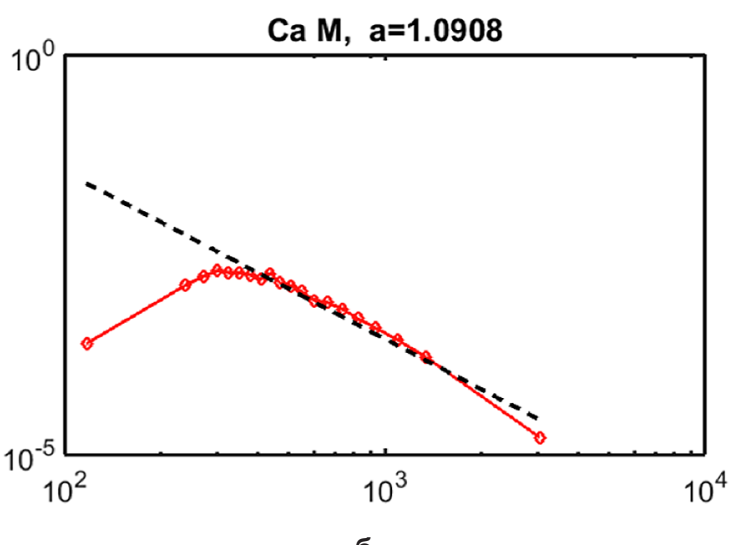

б

Рис. 3. Связь между уровнем Са в эпидермисе в мкг/г (ось ординат) и числом испытуемых (ось абсцисс) у женщин (a) и мужчин (б).

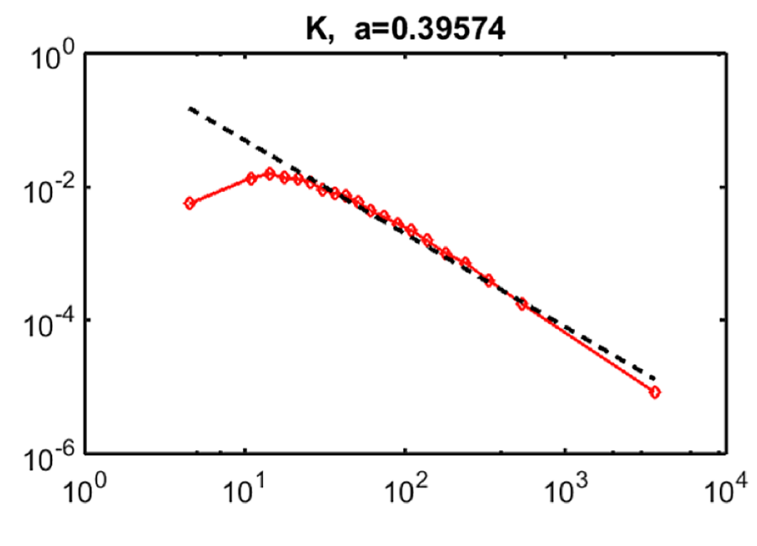

a

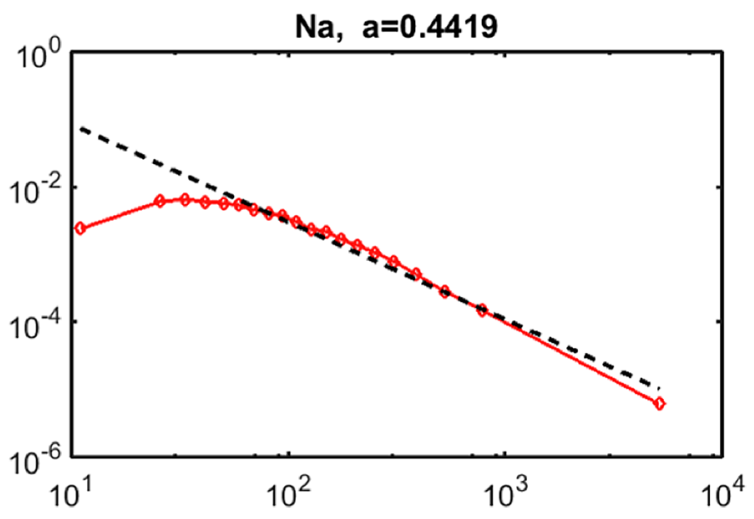

б

Рис. 4. Связь между уровнем калия (а) и натрия (б) в эпидермисе (ось ординат) и числом испытуемых (ось абсцисс). 
Другими словами, в пределах численных значений ЭМ, которые аппроксимируются прямой в двойном логарифмическом масштабе (рис. 3,4 ), имеет место синхронизация (как частный случай критического состояния) не только $\mathrm{Na} / \mathrm{K}-\mathrm{ATPa}$, но, по-видимому, и других мембранных насосов из суперсемейства АТРаз (P-type), ведающих трафиком Сa, Zn и др. металлов $[6,10,12]$.

Основанием для такого допущения могут служить следующие факты.

Проведенный нами ранее корреляционный анализ уровней К и Na в эпидермисе (атомно-эмиссионная спектрометрия) выявил отчётливую линейную зависимость (Pearson) между этими параметрами $\left(r_{\mathrm{K}-\mathrm{Na}}=0,6-0,7\right)$. Исследования проводили у здоровых лиц $(n=947)$ и у ликвидаторов Чернобыльской аварии $(n=954)$, у которых были выявлены признаки окислительного/нитрозативного стресса $[4,8]$. Наличие достоверной К-Nа корреляции свидетельствует о синхронной работе мембранных $\mathrm{Na}^{+} / \mathrm{K}^{+}$-АТРаз, которые в условиях окислительного/нитрозативного стресса «трудятся», по-видимому, с заметно большей интенсивностью. Прямым подтверждением такой возможности служит обнаруженное нами значимое увеличение уровня $\mathrm{Na}$ и K в эпидермисе чернобыльцев, по сравнению с контрольной группой. Косвенным доказательством интенсификации работы $\mathrm{Na}^{+} / \mathrm{K}^{+}$-АТРаз на фоне окислительного/нитрозативного стресса является одновременное и достоверное снижение уровня Са в эпидермисе у чернобыльцев, что, скорее всего, связано с активизацией работы $\mathrm{Na}$ / Ca-обменника (NCX), антипорта для $\mathrm{Ca}^{2+}[1]$.

Дело в том, что Na/K-АТРаза относится к так называемым первичным системам активного транспорта ионов наряду с Са-АТРазой плазматической мембраны, выводящей Са из клетки, и семейством Са-АТРаз эндо- и саркоплазматического ретикулумов (SERCA), закачивающих $\mathrm{Ca}^{2+}$ во внутриклеточные структуры. Вторичный активный транспорт ионов осуществляется за счёт энергии передвижения $\mathrm{Na}^{+}$ в направлении его электрохимического градиента и зависит от эффективной работы Na/K-насоса, который обеспечивает существование этого градиента. Примером вторичного транспорта и является $\mathrm{Na} / \mathrm{Ca}-$ обменник, который выводит один ион кальция $\left(\mathrm{Ca}^{2+}\right)$ за счёт входа в клетку трёх ионов натрия $\left(\mathrm{Na}^{+}\right)$. Поэтому активизация NCX, приводящая к снижению Ca в клетке, может свидетельствовать об интенсивной работе $\mathrm{Na}^{+} / \mathrm{K}^{+}$-АТРаз.

Чтобы убедиться в том, что синхронизация работы мембранных помп распространяется на всё суперсемейство мембранных АТРаз (Р-tyре), мы исследовали тесноту связи (Pearson) между концентрационными значениями K и Zn.

Было интересно выяснить, существуют ли корреляционные взаимоотношения между К и Zn и каков их характер у «чернобыльцев» и здоровых лиц. Полученные результаты не только подтвердили существование K-Zn связи, но и обнаружили различия в её проявлении в сравниваемых группах.

У абсолютного большинства ликвидаторов аварии (88 \%) K-Zn корреляция была негативной и значимой (у 205 человек $r_{\text {к-Zn }}=-0,62 ; p<0,05 ; \mathrm{y}$ 634 человек $\left.r_{\mathrm{K}-\mathrm{Zn}}=-0,41 ; p<0,05\right)$. У $12 \%$ чернобыльцев (115 человек) она не выявлялась $(r=-0,03)$. K-Zn связь отсутствовала $(r=-0,01)$ у 253 здоровых лиц $(26,7 \%)$, у 523 человек (55,2 \%) она была слабо выраженной $\left(r_{\text {к-Zn }}=-0,22 ; p<0,05\right)$ и отчётливо выявлялась $\left(r_{\mathrm{K}-\mathrm{Zn}}=-0,43 ; p<0,05\right)$ лишь у 171 человека (18,1 \%). При этом K-Zn связь при наибольшем |r| (как у чернобыльцев, так и у здоровых лиц) сочеталась со значимо более высоким уровнем K и $\mathrm{Na}$ и более низким уровнем Zn, чем у лиц с отсутствием K-Zn корреляции.

Концентрационные значения металлов в эпидермисе ликвидаторов аварии имели значимые различия с контрольной группой. Результаты спектрометрии эпидермальных клеток, полученные в обеих генеральных совокупностях и в группе здоровых лиц, в зависимости от величины $|r|_{K-Z n}$, представлены в таблице 2.

Анализ металло-лигандного гомеостаза (МЛГ) в эпидермальных клетках у здоровых лиц при крайних значениях $r_{\mathrm{K}-\mathrm{Zn}}(-0,01$ и $-0,43)$ показал, что сдвиги в МЛГ у здоровых с $r_{\mathrm{K}-\mathrm{Zn}}=-0,43$, по сравнению с $r_{\mathrm{K}}$ $\mathrm{zn}=-0,01$, по своей направленности (а в ряде случаев и по величине) аналогичны таковым в общей группе ликвидаторов аварии ( $n=954)$, если сравнивать их с общей контрольной группой $(n=947)$ (табл. 2). Другими словами, 18,1 \% здоровых лиц в этом отношении мало чем отличаются от ликвидаторов аварии.

Вывод, который кажется очевидным, но, тем не менее, требует проверки, это наличие у здоровых лиц $c r_{\text {K-zn }}=-0,43$ окислительного/нитрозативного стресса (возможно, не лучевой природы).

По нашим наблюдениям, как уже говорилось, K-Na связь отличается постоянством, сравнительно высокими значениями коэффициента $r(0,6-0,7)$ и не зависит от размера выборки. В то же время эта корреляция зависит от вида биосубстрата: обнаруживается в волосах и отсутствует в плазме.

Показательно, что выраженная позитивная корреляция между К и $\mathrm{Na}$, которая для всей группы здоровых $(n=947)$ составляла $\left(r_{\mathrm{K}-\mathrm{Na}}=0,64\right)$, практически не менялась $\left(0,64 ; 0,71 ; 0,53 ;\right.$ resp.) при разных $r_{\text {к-Zn }}$. Это может объясняться стабильной (без сбоев) работой мембранного $\mathrm{K}^{+} / \mathrm{Na}^{+}$-насоса у здоровых лиц. Столь же тесной $(r=0,64-0,75) \mathrm{K}-\mathrm{Na} \mathrm{связь} \mathrm{была} \mathrm{у} \mathrm{абсолютного}$ большинства ликвидаторов аварии (в общей группе $\left.r_{\mathrm{K}-\mathrm{Na}}=0,71\right)$, но заметно слабее в подгруппе с $r_{\mathrm{K}-\mathrm{Zn}}=-0,03$ $\left(r_{\mathrm{K}-\mathrm{Na}}=0,31\right)$.

Всё сказанное позволяет допустить, что синхронизация (критичность) может быть присуща нормальной работе всего суперсемейства АТРаз (P-type).

Как уже упоминалось, синхронизация (критическое состояние в терминах СК) принимает вид прямой линии на рисунках 3,4 . Это означает, что в пределах данного интервала численных значений ЭМ, ограниченных прямой, имеется степенная зависимость между уровнем ЭМ и числом индивидов. Существование этой связи и обеспечивает, повидимому, сопряжённость (синхронность) работы мембранных АТРаз. 
БЮЛЛЕТЕНЬ ВСНЦ СО РАМН, 2016, Том 1, № 3(109), Часть II

Таблица 2

Содержание металлов в эпидермальных клетках у здоровых лиц и ликвидаторов Чернобыльской аварии, мкг/г

\begin{tabular}{|c|c|c|c|c|}
\hline \multirow{2}{*}{ Металлы } & \multirow{2}{*}{ Здоровые $(n=947)$} & \multirow{2}{*}{$\begin{array}{c}\text { Ликвидаторы аварии } \\
(n=954)\end{array}$} & \multicolumn{2}{|c|}{ Здоровые ( $n=947)$} \\
\hline & & & $\begin{array}{c}\text { группа, } r_{\text {K-zn }}=-0,01 \\
(n=253)\end{array}$ & $\begin{array}{c}\text { II группа, } r_{\text {к-zn }}=-0,43 \\
(n=171)\end{array}$ \\
\hline $\mathrm{K}$ & $317,7[277,4-361,1]$ & $394,8[365,8-422,4]^{*}$ & $127[82,6-181,9]$ & $894[715,3-1094,8]^{*}$ \\
\hline $\mathrm{Na}$ & $480,9[427,9-542,9]$ & $822,3[757,5-892,4]^{*}$ & $197,8[159,7-245,4]$ & $1233,9[1020,6-1506,5]^{*}$ \\
\hline $\mathrm{Ca}($ жен.) & $1439,6[1348,1-1528,4]$ & $927,4[832,0-1032,8]^{*}$ & $1627,8[1494,6-1770,8]$ & $946,8[788,8-1101,1]^{*}$ \\
\hline $\mathrm{Ca}_{\text {(муж.) }}$ & $620,1[529,1-763,1]$ & $577,6[560,0-601,5]$ & $934,7[755,0-1152,5]$ & $856,9[621,4-1111,6]$ \\
\hline $\mathrm{V}_{\text {(жен.) }}$ & $0,06[0,058-0,07]$ & $0,12[0,10-0,13]^{*}$ & $0,06[0,05-0,07]$ & $0,09[0,08-0,11]^{*}$ \\
\hline $\mathrm{V}_{\text {(муж.) }}$ & $0,09[0,08-0,1]$ & $0,116[0,11-0,12]^{*}$ & $0,06[0,05-0,07]$ & $0,11[0,09-0,13]^{*}$ \\
\hline $\mathrm{Zn}$ & $185,2[181,5-189,3]$ & $165,8[162,5-169]^{*}$ & $204,3[196,0-213,5]$ & $150,7[143,2-158,3]^{*}$ \\
\hline $\mathrm{Cu}$ & $20,7[19,06-22,3]$ & $10,99[10,6-11,4]^{*}$ & $21[18,6-23,5]$ & $16[14,3-18,2]^{*}$ \\
\hline $\mathrm{Cd}$ & $0,05[0,04-0,06]$ & $0,25[0,2-0,3]^{*}$ & $0,03[0,027-0,04]$ & $0,06[0,05-0,08]^{*}$ \\
\hline $\mathrm{Fe}$ & $21,07[19,3-23,1]$ & $23,7[22,4-25,07]^{\star *}$ & $17,8[15,0-21,5]$ & $22,5[20,0-24,9]^{* *}$ \\
\hline $\mathrm{Al}$ & $8,8[8,1-9,5]$ & $20,1[19,3-20,9]^{*}$ & $5,2[4,3-6,2]$ & $12,3[10,8-14,2]^{*}$ \\
\hline $\mathrm{Cr}$ & $0,51[0,48-0,54]$ & $0,9[0,85-0,92]^{*}$ & $0,39[0,35-0,42]$ & $0,72[0,62-0,83]^{*}$ \\
\hline $\mathrm{Pb}$ & $1,1[1,04-1,27]$ & $1,8[1,5-2,2]^{*}$ & $0,95[0,68-1,3]$ & $1,4[1,1-1,6]^{\star *}$ \\
\hline $\mathrm{Li}$ & $0,04[0,03-0,05]$ & $0,06[0,05-0,062]^{*}$ & $0,023[0,02-0,03]$ & $0,05[0,046-0,06]^{*}$ \\
\hline
\end{tabular}

Примечание. величина средней [границы доверительных интервалов (bootstrap-метод)]; различия статистически значимы при ${ }^{*}-p<0,001 ;{ }^{* *}-p<0,05$.

Имеющиеся на графике отклонения от прямой, которые для 그 и К располагаются в начальной части оси абсцисс, а для Са - в конце, т. к. при увеличении $|r|_{K-Z n}$ происходит снижение уровня Са в клетке, соответствуют, согласно теории СК, надкритическому состоянию $(\sigma>1)$.

О переходе в докритическое состояние системы $(\sigma<1)$ можно лишь догадываться по слабо выраженному отклонению от прямой в конечной части оси абсцисс на графиках К и Nа и отчетливо видеть у Ca в начале оси абсцисс. Важно подчеркнуть, что пребывание в до- или надкритическом состоянии означает для системы десинхронизацию её работы.

Интервалы численных значений ЭМ (данные спектрометрии у 947 здоровых лиц (табл. 2)), которые соответствуют асинхронной (до- и надкритическое состояние с $\left.r_{\mathrm{K}-\mathrm{Zn}}=-0,01\right)$ и синхронной работе мембранных насосов (критическое состояние с $r_{\text {к-Zn }}=-0,43$ ) аналогичны данным, полученным в десятикратно большей выборке $(n=10297)$ (рис. 3,4 ).

Это обстоятельство открывает новые возможности для диагностики критических состояний (синхронной работы АТРаз) по содержанию металлов в эпидермисе (спектрометрия). Кроме того, может оказаться полезным (как в клинике, так и в эксперименте) анализ металло-лигандного гомеостаза, который бы учитывал процессы синхронизации/ десинхронизации в работе мембранных помп.

\section{выводы}

Активизация (с участием АФК/АФА) мембранных АТРаз и их нормальное функционирование предполагает синхронный (критический) характер трансмембранного трафика металлов.
Синхронная работа мембранных помп подтверждается отрицательной K-Zn связью (Pearson) и признаками «самоорганизованной критичности» по данным математического анализа.

Полученные результаты указывают на возможную принадлежность гомеостаза электрогенных металлов в эпидермисе к СК-явлениям.

\section{ЛИТЕРАТУРА \\ REFERENCES}

1. Бак П. Теория самоорганизованной критичности. - М.: ЛИБРОКОМ, 2014. - С. 276.

Bak P (2014). Self-organized criticality theory [Teoriya samoorganizovannoy kritichnosti], 276.

2. Закс Л. Статистическое оценивание; пер. с нем. - М.: Статистика, 1976. - С. 598.

Zaks L (1976). Statistical estimation [Statisticheskoe otsenivanie], 598.

3. Argüello JM (2003). Identification of ion-selectivity determinants in heavy-metal transport $\mathrm{P}_{1 \mathrm{~B} \text {-type }}$ ATPases. Journal of Membrane Biology, 195, 93-108.

4. Argüello JM, Eren E, Gonzalez-Guerrero M (2007). The structure and function of heavy metal transport P(1B)-ATPases. BioMetals, 20, 233-248.

5. Axelsen KB, Palmgren MG (1998). Evolution of substrate specificities in P-type ATPase superfamily. Journal of Molecular Evolution, 46, 84-101.

6. Bratic A, Larsson NG (2013). The role of mitochondria in aging. J. Clinical Investigation, Mar 1, 123 (3), 951-957.

7. Kolesnikova LI, Darenskaya MA, Grebenkina LA et al. (2013). The state of the antioxidant status of children of different ages. Voprosy pitaniya, (4), 27-33.

8. Kumerova AO, Lece AG, Skesters AP, Orlikov GA, Seleznev JV, Rainsford KD (2000). Antioxidant defense and 
trace element imbalance in patients with postradiation syndrome: first report on phase I studies. Biological Trace Element Research, 77, 1-12.

9. Lambert AJ et al. (2007). Low rates of hydrogen peroxide production by isolated heart mitochondria associate with long maximum lifespan in vertebrate homertherms. Aging Cell, 6 (5), 607-618.

10. Petukhov VI, Baumane L, Dmitriev EV, Vanin AF (2014). Nitric oxide and electrogenic metals ( $\mathrm{Ca}, \mathrm{Na}, \mathrm{K})$ in epidermic cells. Biochemistry (Moscow) Supplement Series B Biomedical Chemistry, 8 (4), 343-348.
11. Petukhov VI, Baumane LK, Reste ED, Zvagule T, Romanova MA, Shushkevich NI, Sushkova TL, Skavronskii SV, Shchukov AN (2013). Diagnosis of nitrosative stress by quantitative EPR-spectroscopy of epidermal stress. Bulletin of Experimental Biology and Medicine, 154, 734-736.

12. Petukhov VI, Dmitriev EV, Kalvinsh I, Baumane LK, Reste ED, Zvagule T, Skesters AP, Skalny AV (2011). Metal-ligand homeostasis in epidermic cells of Chernobyl accident liquidators. Vitamins \& Trace Elements, 1 (2), 1-8.

Сведения об авторах

\section{Information about the authors}

Петухов Валерий Иванович - доктор медицинских наук, професcop Балтийской международной академии (LV-1003, Латвия, г. Рига, ул. Ломоносова, 4; тел.: (+371) 67100628, (+371) 29786681; e-mail: vip-val@yandex.ru)

Petukhov Valeriy Ivanovich - Doctor of Medical Sciences, Professor of Baltic International Academy (LV-1003, Latvia, Riga, Lomonosov str., 4; tel.: (+371) 67100628, (+371) 29786681; e-mail: vip-val@yandex.ru)

Дмитриев Егор Владимирович - кандидат физико-математических наук, старший научный сотрудник ФГБУН Институт вычислительной математики РАН (e-mail: yegor@inm.ras.ru)

Dmitriev Egor Vladimirovich - Candidate of Physical and Mathematical Sciences, Senior Research Officer of the Institute of Numerical Mathematics RAS (e-mail: yegor@inm.ras.ru)

Баумане Лариса Христофоровна - кандидат химических наук, старший научный сотрудник Института органического синтеза (LV-1006, Латвия, г. Рига, ул. Айзкрауклес, 21; тел. (+371) 67553233; e-mail: Ibaumane@osi.Iv)

Baumane Larisa Khristoforovna - Candidate of Chemical Sciences, Senior Research Officer of Latvian Institute of Organic Synthesis (LV-1006, Latvia, Riga, Ayzkraukles str., 21; tel.: (+371) 67553233; e-mail: Ibaumane@osi.Iv)

Скальный Анатолий Викторович - доктор медицинских наук, профессор, директор Института биоэлементологии (460026, г. Оренбург, пр. Победы, 13; тел.: 8 (3532) 77-70-33; e-mail: skalny@orc.ru)

Skalny Anatoliy Viktorovich - Doctor of Medical Sciences, Professor, Director of Institute of Bioelements (460026, Orenburg, Pobeda av., 13; tel.: +7 (3532) 77-70-33; e-mail: skalny@orc.ru)

Лобанова Юлия Николаевна - научный сотрудник Центра биотической медицины (103064, г. Москва, ул. Земляной Вал, 46/48; тел.: 8 (495) 917-71-21; e-mail: skalny2@orc.ru)

Lobanova Yuliya Nikolaevna - Research Officer of Centre for Biotic Medicine (103064, Moscow, Zemlyanoy Val str., 46/48; tel. +7 (495) 917-71-21; e-mail: skalny2@orc.ru) 